\title{
Mitochondrial stress and activation of PI3K and Akt survival pathway in bladder ischemia
}

This article was published in the following Dove Press journal:

Research and Reports in Urology

10 June 2017

Number of times this article has been viewed

Jing-Hua Yang'

Mike B Siroky'

Subbarao V Yalla ${ }^{2}$

Kazem M Azadzoi ${ }^{3,4}$

'Department of Urology, VA Boston Healthcare System, Boston University

School of Medicine, ${ }^{2}$ Department of Urology, VA Boston Healthcare System, Harvard Medical School, ${ }^{3}$ Department of Urology, ${ }^{4}$ Department of Pathology, VA Boston Healthcare System, Boston University School of Medicine, Boston, MA, USA
Correspondence: Kazem M Azadzoi Department of Urology, VA Boston Healthcare System, Building IA, Room 317 (15I), I50 South Huntington Avenue,

Tel +l 8573645602

Email kazadzoi@bu.edu Boston, MA 02130 , USA

Purpose: Detrusor overactivity contributes to bothersome constellation of lower urinary tract symptoms (LUTS) in men and women as they age. However, the underlying mechanisms of nonobstructive detrusor overactivity and LUTS remain largely unknown. Growing evidence suggests that ischemia may be an independent factor in the development of non-obstructive bladder dysfunction. Our goal was to determine the effects of ischemia on detrusor function and voiding behavior and define redox-mediated cellular stress and cell survival signaling in the ischemic bladder.

Materials and methods: Male Sprague Dawley rats were randomly divided into treatment $(n=8)$ and control $(n=8)$ groups. In the treatment group, iliac artery atherosclerosis and chronic bladder ischemia were induced. At 8 weeks after bladder ischemia, voiding patterns were examined in metabolic cages, cystometrograms were recorded in conscious animals, and then bladder blood flow was measured under general anesthesia. Bladder tissues were processed for assessment of transcription factors, markers of cellular and mitochondrial stress, mitochondrial respiration, and cell survival signaling pathway.

Results: Atherosclerotic occlusive disease spread from the common iliac arteries to the internal iliac and vesical arteries and produced sustained bladder ischemia. Studies in metabolic cages showed increased micturition frequency and decreased voided volume in bladder ischemia. Conscious cystometrograms produced consistent data showing significant increase in micturition frequency and decreased voided volume and bladder capacity. Voiding behavior and cystometric changes in bladder ischemia were associated with significant decrease in DNA binding activity of Nrf2, significant increase in cellular levels of stress protein Hsp70 and mitochondrial stress protein GRP75, and significant decrease in mitochondrial oxygen consumption and upregulation of PI3K and Akt expression.

Conclusion: Chronic bladder ischemia may be a mediating variable in the development of detrusor overactivity in the non-obstructive bladder. The mechanism may involve ischemiainduced cellular stress, Nrf2 functional deficit, depression of mitochondrial respiration, and upregulation of PI3K/Akt cell survival signaling pathway.

Keywords: atherosclerosis, blood flow, redox, cellular stress, detrusor overactivity, survival signaling

\section{Introduction}

Lower urinary tract symptoms (LUTS) such as urgency, frequency, incontinence, nocturia, intermittency, slow stream, hesitancy, postmicturition dribble, and the sensation of incomplete bladder emptying are bothersome constellation of voiding symptoms affecting both men and women as they age. The incidence of LUTS increases with aging, and our understanding of the mechanisms for LUTS in the elderly is evolving. The mechanism of detrusor overactivity and LUTS in patients with non-obstructed 
bladder remains largely unknown. Aging-associated bladder wall changes predisposing to the development of detrusor overactivity and LUTS remain essentially unknown. While most cases of LUTS in male are attributed to bladder outlet obstruction due to benign prostatic enlargement (BPE), in approximately one third to more than one half of cases, LUTS are not associated with BPE or bladder outlet obstruction. ${ }^{1-4}$ Furthermore, symptom questionnaires designed to document LUTS in women provide identical scores to those of their age-matched male counterparts. ${ }^{5,6}$ Urodynamic studies have shown that aging-associated changes in maximum flow rate, voided volume, and post-void residual volume are similar in both sexes. ${ }^{5,6}$ These observations suggest that aging-related sex-independent mechanisms involving vasculature, nerves, smooth muscle cells, and epithelium may be responsible for detrusor overactivity in the non-obstructive bladder.

A recently described mechanism of detrusor overactivity and LUTS in the elderly is bladder ischemia due to pelvic atherosclerotic disorders. First reported in experimental models and later in patients, pelvic arterial atherosclerosis and bladder ischemia were shown to be important factors in detrusor overactivity and LUTS. Animal studies showed that structural and functional consequences of bladder ischemia depend on the severity and duration of arterial obstructive disease. ${ }^{7,8}$ Short-term moderate ischemia altered muscarinic and purinergic receptor expression and led to detrusor overactivity, while prolonged severe ischemia impaired the smooth muscle energy resources, activated inflammatory pathways, and led to fibrosis and degeneration of the smooth muscle cells and nerve fibers. ${ }^{9-11}$ Changes in prolonged ischemia appeared to involve free radicals and redox-mediated upregulation of cytokines and inflammatory eicosanoids and leukotrienes. ${ }^{9-11}$

The goal of the present study was to examine detrusor function, measure the levels of cellular stress response proteins, and assess mitochondrial respiration rate and the status of redox-induced cell survival signaling in chronic bladder ischemia.

\section{Methods}

\section{Animal model of bladder ischemia}

Animal care and experimental protocols were in accordance with the guidelines and approval of the VA Boston Healthcare System Institutional Animal Care and Use Committee. Adult male Sprague Dawley rats were randomly divided into treatment $(n=8)$ and sham control $(n=8)$ groups. The treatment group received a $2 \%$ cholesterol diet for 2 weeks, and then balloon dilation of the iliac arteries was performed using a $2 \mathrm{~F}$ Fogarty arterial embolectomy catheter (Edwards Lifesciences
Corp., Irvine, CA, USA). The goal of arterial ballooning was to disrupt endothelial cells and induce atherosclerotic occlusive disease, as previously described. ${ }^{4-7}$ After the procedure, the animals received $2 \%$ cholesterol diet until studied. The sham group underwent similar procedures without arterial ballooning and received a regular diet. After 8 weeks, the animals underwent in vivo examination, and then bladder tissues were processed for analysis.

\section{Assessment of voiding patterns}

Micturition patterns of the animals were examined for $24 \mathrm{~h}$ using the metabolic cage system. Total number of voids per $24 \mathrm{~h}$ and voided volume were continuously recorded via a fluid collector attached to the metabolic cage and connected to a digital balance below the metabolic cage. The balance was connected to a computer with data acquisition. Both voiding frequency and mean voided volume were continuously recorded. The animal's drinking bottles were filled with a known volume of water, and water intake per $24 \mathrm{~h}$ was measured.

\section{Cystometrograms}

To perform conscious cystometry, the animals underwent a surgical procedure as described below, and then cystometrograms were obtained 3 days after the procedure. The animals were anesthetized with inhalation of $1-2 \%$ isoflurane mixed with oxygen, and then the bladder was exposed and a polyethylene PE-50 catheter was inserted into the dome, secured with a purse, string suture, routed subcutaneously to the back of the animal and then coiled and stored in a skin pouch. After 3 days, the coiled catheter was removed and connected to a pressure transducer. The animal was placed in a small animal cystometry station, and conscious cystometrograms were obtained. To measure voided urine volume, an analytical balance was placed under the cage. For cystometry, the bladder was emptied, and then saline was infused via a syringe pump at a rate of $150 \mu \mathrm{L} \mathrm{min}{ }^{-1}$. Bladder capacity was determined by measuring the total amount of saline infused into the bladder at the time when micturition commenced.

\section{Measurement of bladder blood flow}

After completion of the conscious cystometry, the animals were anesthetized, an abdominal incision was made to expose the bladder, and then bladder blood flow was measured under general anesthesia as previously described..$^{7-10}$ In brief, a laser Doppler probe was inserted into the bladder wall and connected to a blood flow meter (Transonic Systems, Inc., Ithaca, NY, USA). Bladder blood flow was recorded at a constant intravesical volume of $0.8 \mathrm{~mL}$. 


\section{Enzyme-linked immunosorbent assay (ELISA)-based assessment of Nrf2 activity and cellular stress}

DNA-binding activity of Nrf2 in the nuclear extracts obtained from the ischemic and control bladder tissues was examined using the TransAM Nrf2 ELISA kit (Active Motif Inc., Carlsbad, CA, USA). In brief, nuclei were separated from the cytoplasm in each sample using standard centrifugation technology, and then nuclear extracts from each sample in triplicate were incubated in a 96-well plate according to the manufacturer's instructions. Optical density was read using a plate reader at $450 \mathrm{~nm}$ and standardized as percentage change versus control. Cellular stress response to ischemia and hypoxia was determined by measuring the levels of the stress response protein namely Hsp70 using ELISA kit (Enzo Life Sciences, Farmingdale, NY, USA) according to manufacturer's instructions. Each sample was run in duplicate. Optical density was read using a plate reader at $450 \mathrm{~nm}$, and the data were standardized as nanogram per milliliter according to the calibration curve obtained from serial dilutions of Hsp70 standard.

\section{Western blotting of mitochondrial stress protein $\mathrm{mtH} s \mathrm{sp} 70$ or GRP75}

For Western blotting of the mitochondrial stress protein namely mtHsp70 or GRP75, bladder tissue samples were harvested and stored at $-80^{\circ} \mathrm{C}$ until use. Tissue lysates were obtained, centrifuged, and supernatants were collected. Protein was separated with sodium dodecyl sulfatepolyacrylamide gel electrophoresis (SDS-PAGE) and transferred to polyvinylidene difluoride filter membranes (Millipore, Bedford, MA, USA). The membranes were incubated overnight with either GRP75 or $\beta$-actin antibody (Cell Signaling, Beverly, MA, USA) at $4^{\circ} \mathrm{C}$. The membrane was then incubated with secondary antibody for $2 \mathrm{~h}$ and scanned with Typhoon 8600 imager (GE Healthcare, Pittsburg, PA, USA). Protein expression was quantitated by densitometry using ImageJ software.

\section{Measurement of mitochondrial oxygen consumption}

Bladder tissue samples were washed, minced in phosphate-buffered saline, and homogenized with a glass homogenizer. The lysates were centrifuged at $1,000 \times g$ for $5 \mathrm{~min}$ at $4^{\circ} \mathrm{C}$, and then the supernatant was collected and centrifuged at $12,000 \times g$ for $10 \mathrm{~min}$ at $4 \mathrm{C}$; this step was performed twice. The final mitochondrial pellet was washed, centrifuged, resuspended, added to respiration buffer, and processed for oxygen consumption measurement using a Clark electrode (model 949, Strathkelvin Instruments, Motherwell, UK) in a water-jacketed glass reaction vessel (Mitocell MT200).

\section{Assessment of cell survival signaling}

The goal was to determine whether bladder stress responses to ischemia involve the PI3K and Akt cell survival pathway. Ischemic and control bladder tissues were homogenized and centrifuged. Equal protein concentrations from each sample were processed for SDS-PAGE analysis. The membrane was washed, blocked, and then incubated with either anti PI3K (Abcam, Cambridge, MA, USA), Akt1 (Santa Cruz, Dallas, TX, USA), or beta-actin (Santa Cruz) antibody. After this, samples were washed, incubated with the secondary antibody, and then washed in Tris-buffered saline. Protein expression was determined by densitometry and analyzed using the ImageJ program.

\section{Statistical analysis}

Data are expressed as mean \pm standard error of the mean. Statistically significant differences in the ischemic group versus sham control were determined using Student's $t$-test. Significant changes were determined at $p<0.05$ level.

\section{Results \\ Effects of bladder ischemia on voiding patterns and cystometrograms}

Histology of blood vessels showed that atherosclerotic occlusive disease spreads from the site of arterial ballooning to smaller arteries involving the internal iliac and vesical arteries. Arterial atherosclerosis significantly decreased bladder blood flow (milliliter per minute per $100 \mathrm{~g}$ tissue) to $4.6 \pm 0.6$ versus $10.2 \pm 1.1$ in sham controls $(p=0.003)$. Bladder ischemia altered voiding patterns and cystometrograms (Figure 1). Micturition frequency per $24 \mathrm{~h}$ of $17.6 \pm 1.3$ in animals with bladder ischemia was significantly greater in comparison with $11.7 \pm 1.2$ in the sham control group ( $p=0.046)$. Voided volume $(\mathrm{mL})$ of $0.86 \pm 0.08$ in animals with bladder ischemia was significantly less in comparison with $1.30 \pm 0.13$ in the sham control group (0.032). Baseline and threshold bladder pressures $\left(\mathrm{cm} \mathrm{H}_{2} \mathrm{O}\right)$ of $11.8 \pm 1.6$ and $21.5 \pm 1.7$, respectively, in animals with bladder ischemia were similar to $10.4 \pm 1.1$ and $19.6 \pm 1.9$, respectively, in sham controls. The ischemic bladder capacity $(\mathrm{mL})$ of $1.20 \pm 0.13$ was significantly less in comparison with $1.66 \pm 0.11$ in the sham control group ( $p=0.022)$. 


\section{Conscious cystometry}
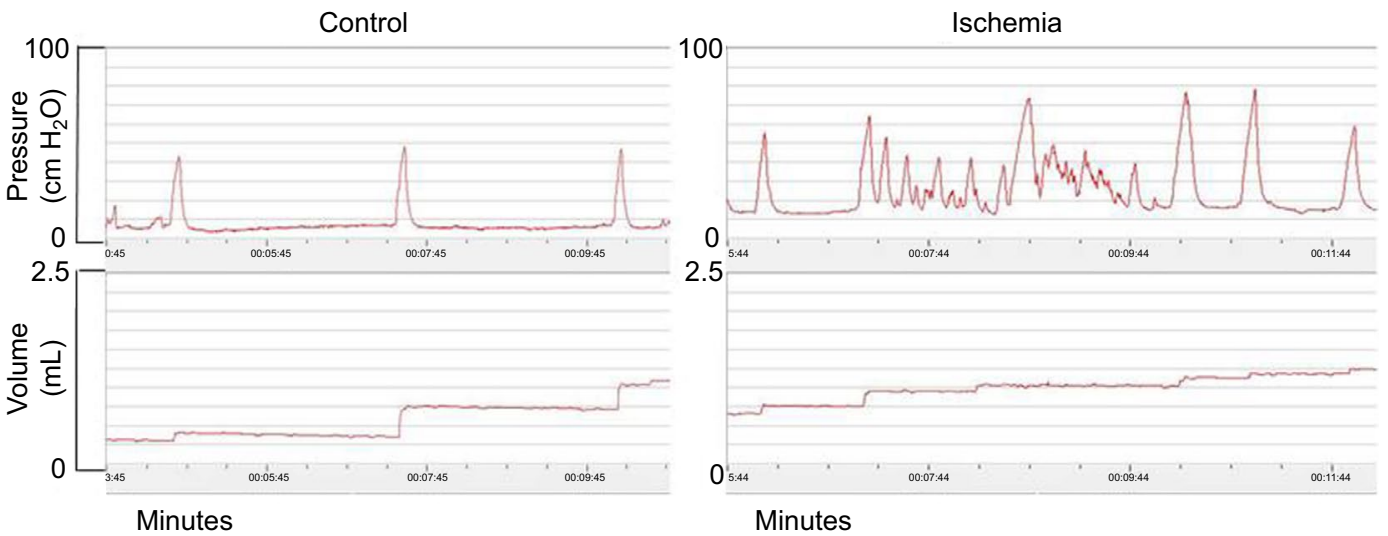

Figure I Conscious cystometric measurements with infusion of saline at $150 \mu \mathrm{L} \mathrm{min}{ }^{-1}$ are shown in rats with bladder ischemia versus sham control.

Notes: Bladder pressure changes are shown in upper panels, and voided volumes are shown in the lower panels. Changes in voiding bladder contractions, micturition frequency, and voided volumes in rats with bladder ischemia are consistent with detrusor overactivity.

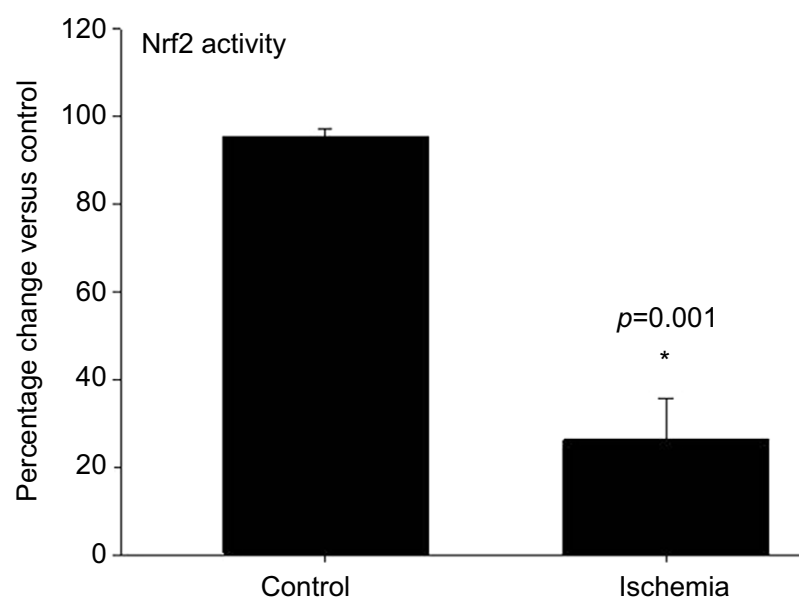

Figure 2 ELISA of Nrf2 activity in the ischemic and control bladder samples. Notes: Bladder ischemia caused a significant decrease in Nrf2-DNA binding activity. *Represents significant difference versus control.

Abbreviation: ELISA, enzyme-linked immunosorbent assay.

\section{Impairment of Nrf2-DNA binding activity}

Bladder ischemia impaired nuclear translocation and activation of the redox-sensitive factor Nrf2. Ischemia caused a significant decrease in DNA binding activity of Nrf2, suggesting cytoplasmic sequestration of Nrf2 and lack of cellular antioxidant defense via $\mathrm{Nrf} 2$ antioxidant response element signaling pathway (Figure 2).

\section{Mitochondrial stress in bladder ischemia} Impairment of Nrf2-DNA binding activity in bladder ischemia was associated with markers of ischemia- and redoxinduced cellular stress and accumulation of cellular stress proteins. ELISA showed a significant increase in cellular levels of stress protein Hsp70 (Figure 3). Western blotting showed significant increase in the levels of mitochondrial stress protein MtHsp70, namely GRP75, suggesting mitochondrial stress in bladder ischemia (Figure 4).

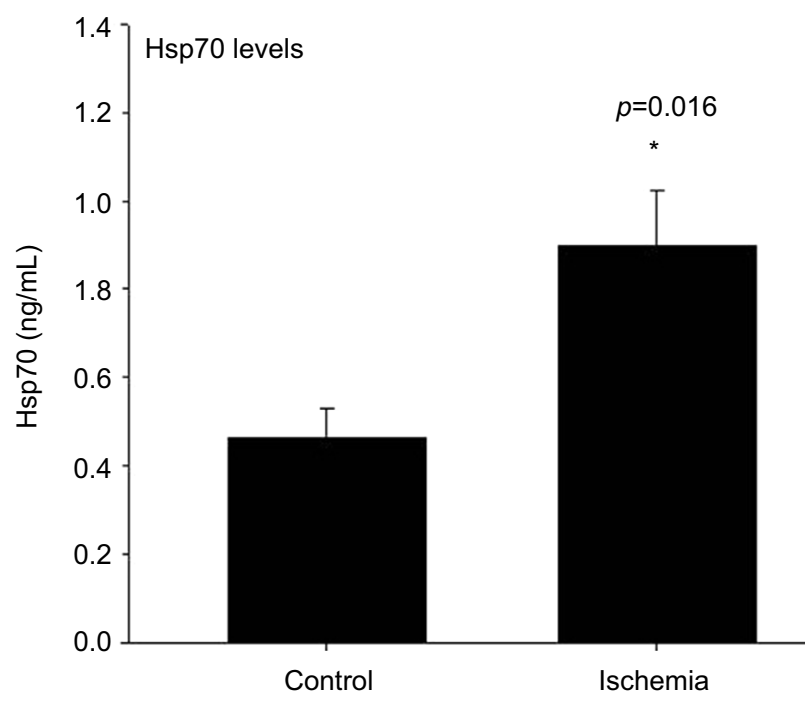

Figure 3 ELISA of cellular stress protein Hsp70 in the ischemic and control bladder samples.

Notes: Bladder ischemia significantly increased Hsp70 levels, suggesting cellular stress. *Represents significant difference versus control.

Abbreviation: ELISA, enzyme-linked immunosorbent assay.

\section{Depression of mitochondrial respiration}

Mitochondrial stress in bladder ischemia was associated with marked decrease in mitochondrial respiration rate characterized by significant decrease in mitochondrial oxygen consumption in the ischemic bladder tissues versus control samples (Figure 5).

\section{Activation of cell survival signaling}

Increased levels of stress proteins and decreased mitochondrial respiration in bladder ischemia were associated with upregulation of cell survival signaling pathways. Western blotting showed significant increases in PI3K (Figure 6) and phosphorylated Akt (Figure 7) expressions in the ischemic 
Mitochondrial stress in bladder ischemia

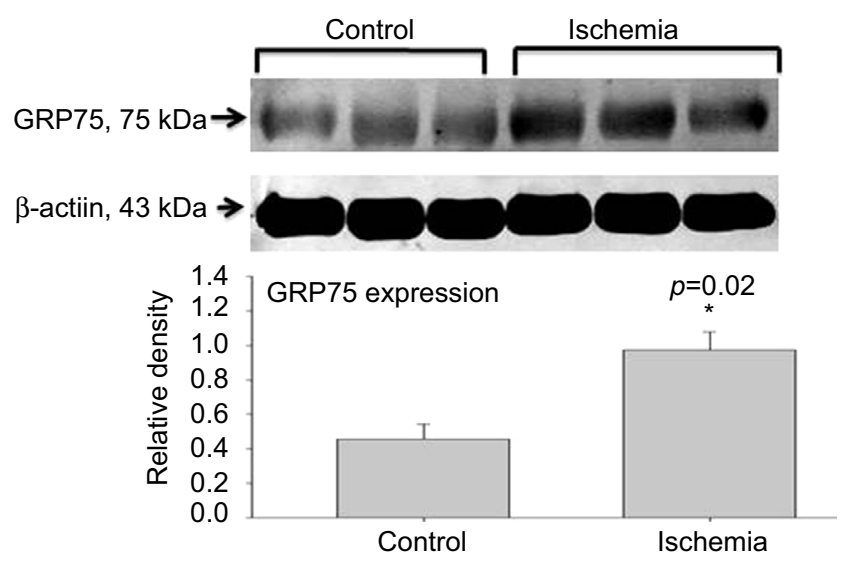

Figure 4 Western blotting of mitochondrial stress protein GRP70 in the ischemic and control bladder samples.

Notes: Bladder ischemia caused a significant increase in cellular levels of GRP70, suggesting mitochondrial stress. *Represents significant difference versus control.

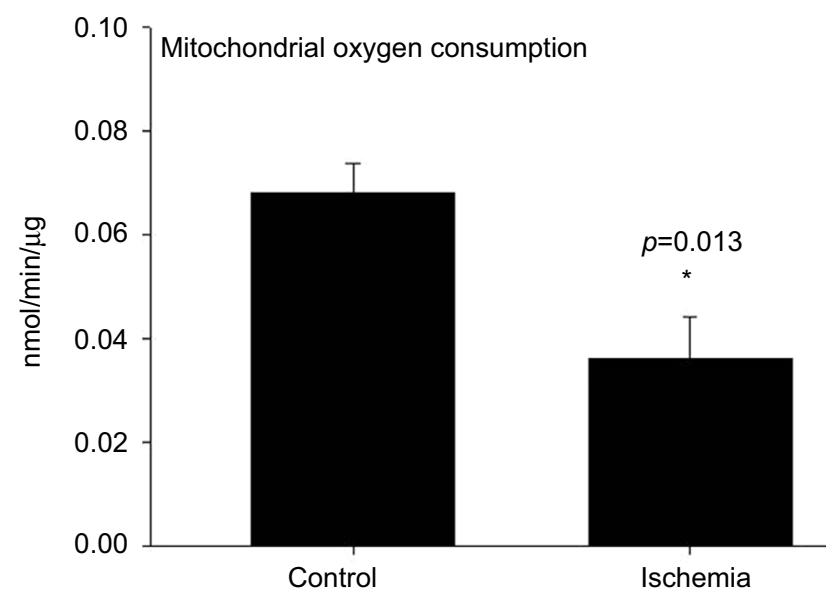

Figure 5 Mitochondrial oxygen consumption rate in the ischemic and control bladder samples.

Notes: Bladder ischemia caused a significant decrease in mitochondrial oxygen consumption rate, suggesting depression of mitochondrial respiration in ischemia. *Represents significant difference versus control.

bladder tissues, suggesting cell danger and hypoxia- and redox-induced survival signaling.

\section{Discussion}

Increased micturition frequency, decreased voided volume and cystometric parameters in the rat model of bladder ischemia are consistent with detrusor overactivity. Our previous studies have shown that lack of perfusion, accumulation of metabolic waste, and production of free radicals in bladder ischemia initiate a cascade of molecular responses and lead to cell danger signals..$^{7-10}$ Bladder responses to ischemia resulted in sensitization of smooth muscle cells to contractile stimuli and increased detrusor activity. ${ }^{7-10}$ Our present study suggests that redox-mediated cellular stress, differential translocation
Survival signaling in bladder ischemia

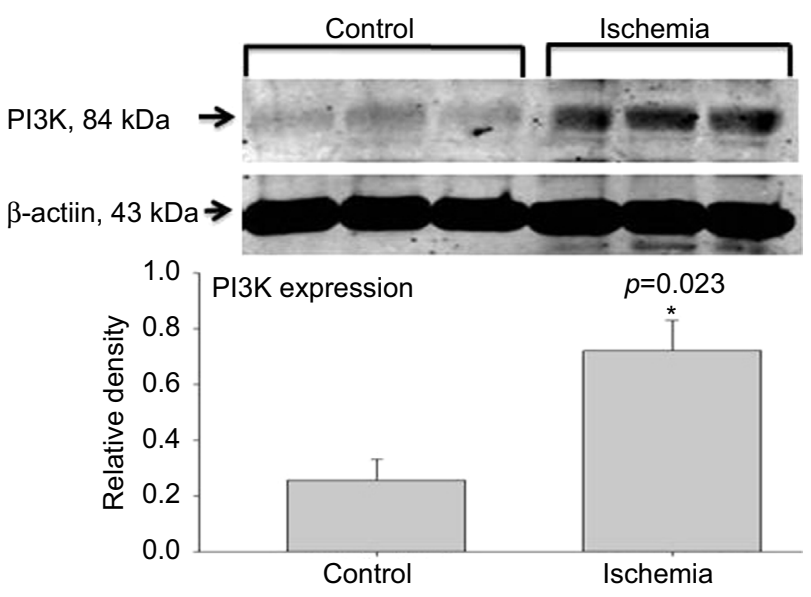

Figure 6 Western blotting of PI3K in the ischemic and control bladder samples. Notes: Ischemia caused a significant increase in bladder PI3K expression, suggesting cell survival signaling. *Represents significant difference versus control.

\section{Survival signaling in bladder ischemia}

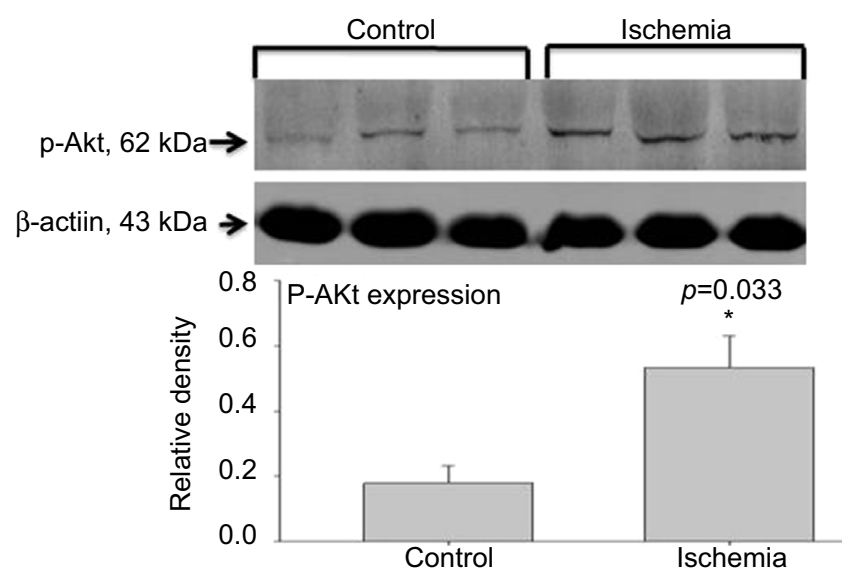

Figure 7 Western blotting of Akt in the ischemic and control bladder samples. Notes: Ischemia caused a significant increase in bladder Akt expression, suggesting cell survival signaling. *Represents significant difference versus control.

of transcription factors, mitochondrial dysfunction, and activation of cell survival pathways may contribute to alterations of smooth muscle contractile activity and detrusor function in bladder ischemia. Cell danger signals and cellular stress elements in ischemia stimulate the nerves, receptors, and smooth muscle cells to develop protective measures to support survival. These cellular stress responses are thought to influence neurotransmission discharge and compromise receptor expression and smooth muscle contractile activity.

Basic research evidence of detrusor overactivity and voiding dysfunction in bladder ischemia has been supported by clinical observations of a close relationship between human lower urinary tract ischemia and LUTS. ${ }^{12-19}$ In clinical studies, lower urinary tract blood flow has been measured and bladder 
ischemia documented in elderly patients with LUTS. ${ }^{12-19}$ Decreased bladder blood flow significantly correlated with LUTS in both men and women. ${ }^{12-17}$ The degrees of pelvic atherosclerosis significantly correlated with the severity of LUTS in elderly patients. ${ }^{12,16}$ It was shown that human bladder blood flow decreases with aging and decreased bladder blood flow significantly correlates with reduced compliance. ${ }^{14}$ In men, LUTS improvement with alpha adrenoceptor blockers was associated with significant increase in bladder blood flow. ${ }^{17}$ Regulation of TL1A and DR3 by bladder ischemia and potential roles of TL1A and DR3 in bladder inflammation were documented in the elderly patients. ${ }^{19}$ Dutasteride was shown to reduce overactive bladder symptoms by increasing blood flow and improving bladder ischemia. ${ }^{20}$

Ischemia, in general, is a condition that occurs due to disruption of blood supply to a particular tissue or organ, which cuts the supply of oxygen, glucose, and nutrients leading to dysfunction and a cascade of inflammatory events that end with cell death. Chronic ischemia and hypoxia lead to inexorable accumulation of noxious radicals and oxidative damage to biomolecules vital for proper cellular function. The oxidative process is fundamentally haphazard and uncontrollable and ultimately interfaces with cellular stress and cell danger signaling. Bladder, a contractile and highly metabolic organ, has high oxygen consumption. Functional and metabolic properties of the bladder make it sensitive to oxygen deficiency and increase its vulnerability to ischemic insult. Stress responses to ischemia involve a cascade of cellular events involving energy failure, mitochondrial damage, free radical production, oxidative stress, and inflammation that ultimately lead to degeneration of smooth muscle cells and nerve fibers. ${ }^{7-11}$ Cell senses varieties of stress conditions via different sensors. Cellular stress activates survival signaling pathways to signal cell danger and initiate defensive responses to protect cell structure and function. Cellular stress response is the homeostatic mechanism, which directs cells to adapt to various stress cues such as ischemia, hypoxia, and redox.

Our present study suggests that chronic ischemia impairs Nrf2-DNA binding activity in the bladder. Lack of Nrf2 activity and subsequent decrease in cellular antioxidant defense capacity may contribute to cellular and mitochondrial stress and increased levels of stress proteins Hsp70 and GRP75 in bladder ischemia. Upregulation of mitochondrial stress protein GRP75 was associated with impairment of mitochondrial respiration. These cellular responses to ischemia may play a role in cell survival signaling via PI3K/Akt pathway to promote function and preserve subcellular structures.
The PI3K/Akt signaling pathway plays a critical role in cell danger signals in a wide range of cellular stress conditions including ischemia and redox. ${ }^{21,22}$ Activation of PI3K/Akt pathway is a signal transduction that promotes survival and growth in response to integrating stress signals through the stress sensors. ${ }^{21,22}$ Survival signaling via PI3K/Akt pathway can be activated exogenously by growth factors and cytokines and endogenously by the constitutively active regulatory proteins. $^{21,22}$ Phosphorylated AKT enhances survival and inhibits apoptosis by phosphorylation and inactivation of prospective target proteins.

Inhibition of Nrf2 activity by ischemia may be considered as the starting point for cellular stress and survival signaling in the bladder. Lack of Nrf2-DNA binding activity in bladder ischemia may suggest blockade of Nrf2 translocation to the nucleus and potential sequestration of Nrf2 in the cytoplasm. The Keap1-Nrf2 system was identified as a leading regulator of oxidative stress responses and cell survival against oxidative stress. ${ }^{23}$ The antioxidant defense and cell survival properties of Nrf2 are thought to be mediated by mitochondria. ${ }^{24}$ This concept is supported by the evidence of various types of cross talk between the Keap1-Nrf2 system and mitochondria. ${ }^{24,25} \mathrm{Nrf2}$ is ubiquitously expressed but its expression is relatively higher in organs with contractile function that interface frequent blood flow and oxygen tension changes. $\mathrm{Nrf} 2$ plays an important role in removing deleterious free radicals by upregulating a set of detoxification enzymes and antioxidant proteins. ${ }^{23}$ In non-stressed cells, Nrf2 plays little role and is rapidly degraded by the Keap1-mediated ubiquitin proteasome system. ${ }^{23}$ In ischemic tissues and oxidative stress conditions, Nrf2 translocates to the nucleus where it binds to antioxidant responsive elements and activates the expression of target genes, including glutathione S-transferases or antioxidant proteins such as HO-1 and thioredoxin. ${ }^{23}$ Oxidative stress causes the oxidation of cysteine residues in Keap1, resulting in a conformational change in Keap1 leading to inhibition of Nrf2 ubiquitination and degradation.

Impairment of Nrf2-DNA binding activity in bladder ischemia was associated with increased levels of mitochondrial stress proteins and significant decrease in mitochondrial respiration. Mitochondria generate energy by cellular respiration and serve as an integrative platform for a cascade of intracellular signaling pathways that mediate inflammatory responses and degenerative mechanisms. ${ }^{26,27}$ Mitochondria are also the major source of free radicals and play an integral role in the determination of cell survival and degeneration upon exposure to stress. ${ }^{26}$ Most of the mitochondrial proteins are encoded in the nuclear genome. Therefore, 
communication between the mitochondria and nucleus is essential for maintaining cellular homeostasis against free radical incursion and stress conditions. ${ }^{27} \mathrm{Nrf} 2$ also plays a role in mitochondrial biogenesis. It was shown that Nrf2 mediates mitochondrial biogenesis and mitochondrial biogenesis was impaired in Nrf2 knockout mice. ${ }^{28}$ Exposure to mild-to-moderate hypoxic conditions produced excitotoxicity and mitochondria-mediated degeneration of hippocampal neurons, leading to cognitive impairment. ${ }^{29}$ Another study showed that nitric oxide (NO) activates mitochondrial biogenesis in an Nrf2-dependent manner. ${ }^{30}$

Activation of survival signaling via PI3K/Akt pathway in response to cellular stress imposes enormous burden on mitochondria to produce energy and maintain function. Upregulation of PI3K and Akt and subsequent activation of cell survival signaling pathways stimulate the mitochondria to produce energy for survival and protect cells from redox under the ischemic conditions. Survival signaling also influences neural and smooth muscle cell physiological function leading to hypersensitivity and irregular contractile activity. ${ }^{31,32}$ Studies of ischemic heart, gastrointestinal system, and brain have documented activation of survival signaling events with adverse impact on neural reactivity, receptors function, and smooth muscle cell contractility. ${ }^{33}$ Survival responses were shown to signal nutrient deficiency and hypoxia and regulate defensive reactions against free radicals under the hypoxic and oxidative stress conditions. ${ }^{31-33}$

Upregulation of the serine-threonine Akt may be a defensive mechanism that signals cell danger due to hypoxia and redox and may play a key role in molecular responses and cellular and subcellular reactions in bladder ischemia. Akt is the main downstream effector of PI3K, and its activation is initiated by membrane recruitment via interaction with phosphatidylinositol 3,4,5-trisphosphate. After membrane anchoring, Akt is sequentially activated by phosphorylation mechanisms that result in its translocation from the plasma membrane to intracellular compartments, including the cytoplasm and nucleus, where it phosphorylates a variety of molecules and activates downstream pathways. ${ }^{34}$ Another important target of PI3K/Akt signaling is the class $\mathrm{O}$ of FoxO transcription factors. The FoxO family may play a key role in cellular degenerative processes under the ischemic and redox conditions as they are known to increase the expression of pro-apoptotic genes. ${ }^{35}$ FoxO transcriptional activity and its subcellular localization are dependent on the phosphorylation of threonine 24, serine 256, and serine 319 by Akt. It was also shown that chronic cellular stress and enduring phosphorylation and superphysiological activation of Akt may increase oxidative stress levels and contribute to oxidative injury in persisting redox conditions. The mechanism is thought to involve Akt-dependent upregulation of oxidative phosphorylation and oxygen consumption. Another possibility is that overactivated Akt could lead to sustained inhibition of FoxO transcription factors, which normally increases the expression of antioxidant elements such as superoxide dismutase and catalase. These observations suggest that ischemia- and redox-induced cellular stress and subsequent activation of cell survival pathway may provide temporary protection against free radicals and cytotoxic elements. In prolonged ischemia, cellular defensive mechanisms and survival pathways fail to protect cellular function and structural integrity due to persistent hypoxia, accumulation of metabolic waste, and unchecked production of noxious free radicals leading to inflammatory and degenerative responses.

\section{Conclusion}

Clinical research suggests a close correlation between vascular risk factors, bladder ischemia, and the development of LUTS in elderly patients. The severity of LUTS in elderly patients was shown to correlate with the number of vascular risk factors and decreased blood flow to the bladder. Our present study shows that chronic bladder ischemia in rats produces voiding behavior and cystometric changes consistent with detrusor overactivity. Functional changes in bladder ischemia were associated with decreased Nrf2-DNA binding activity, impairment of mitochondrial respiration, markers of cellular and mitochondrial stress, and activation of cell survival signaling via PI3K/Akt pathway. These observations may suggest the involvement of ischemia-induced cellular stress and survival pathway in smooth muscle instability and the development of detrusor overactivity in the non-obstructive bladder.

\section{Acknowledgment}

This research was supported by Grant BLR\&D MERIT 1I01BX001428 from the US Department of Veterans Affairs.

\section{Disclosure}

The authors report no conflicts of interest in this work.

\section{References}

1. Bosh JLHR, Kranse R, Mastright R, Schröder FH. Reasons for the weak correlation between prostate volume and urethral resistance parameters in patients with prostatism. $J$ Urol. 1995;153(3 pt 1):689.

2. Yalla SV, Sullivan MP, Lecamwasam HS, DuBeau CE, Vickers MA, Cravalho EG. Correlation of American Urological Association symptom index with obstructive and nonobstructive prostatism. J Urol. 1995;153(3 pt 1):674.

3. Dørflinger T, England DM, Madsen PO, Bruskewitz RC. Urodynamic and histological correlates of benign prostatic hyperplasia. J Urol 1988;140(6): 1487. 
4. Fultz NH, Herzog AR. Epidemiology of urinary symptoms in the geriatric population. Urol Clin North Am. 1996;23(1):1-10.

5. Madersbacher S, Pycha A, Schatzi G, Mian C, Klingler CH, Marberger $\mathrm{M}$. The aging lower urinary tract: a comparative urodynamic study of men and women. Urology. 1998;51(2):206.

6. Lepor H, Machi G. Comparison of AUA symptom index in unselected males and females between fifty-five and seventy-nine years of age. Urology. 1993;42(1):36.

7. Azadzoi KM, Tarcan T, Kozlowski R, Krane RJ, Siroky MB. Overactivity and structural changes in the chronically ischemic bladder. $J$ Urol. 1999;162(5): 1768-1778.

8. Zhao Z, Azad R, Yang JH, Siroky MB, Azadzoi KM. Progressive changes in detrusor function and micturition patterns with chronic bladder ischemia. Investig Clin Urol. 2016;57(4):249-259.

9. Su N, Choi HP, Yang J-H, et al. Quantitative proteomic analysis of differentially expressed proteins and downstream signaling pathways in chronic bladder ischemia. J Urol. 2016;195(2):515-523.

10. Azadzoi KM, Radisavljevic ZM, Golabek T, Yalla SV, Siroky MB. Oxidative modification of mitochondrial integrity and nerve fiber density in the ischemic overactive bladder. $J$ Urol. 2010;183(1):362-369.

11. Azadzoi KM, Yalla SV, Siroky MB. Human bladder smooth muscle cell damage in disturbed oxygen tension. Urology. 2011;78(4): 967.e9-967.e15.

12. Takahashi N, Shishido K, Sato Y, et al. The association between severity of atherosclerosis and lower urinary tract function in male patients with lower urinary tract symptoms. Low Urin Tract Symptoms. 2012;4(1):9-13.

13. Batista JE, Wagner JR, Azadzoi KM, Krane RJ, Siroky MB. Direct measurement of blood flow in the human bladder. J Urol. 1996;155(2): 630-633.

14. Kershen RT, Azadzoi KA, Siroky MB. Blood flow, pressure and compliance in the male human bladder. $J$ Urol. 2002;168(1):121-125.

15. Germar-Michael P, Michael M, Eberhard S, et al. Association of lower urinary tract symptoms and chronic ischaemia of the lower urinary tract in elderly women and men: assessment using colour Doppler ultrasonography. BJU Int. 2008;102(4):470-474.

16. De EJ, Hou P, Estrera A, et al. Pelvic ischemia is measurable and symptomatic in patients with coronary artery disease: a novel application of dynamic contrast-enhanced magnetic resonance imaging. J Sex Med. 2008;5(11):2635.

17. Pinggera GM, Mitterberger M, Pallwein L, et al. Alpha-blockers improve chronic ischaemia of the lower urinary tract in patients with lower urinary tract symptoms. BJU Int. 2007;101(3):319-324.

18. Kapoor H, Gupta E, Sood A. Chronic pelvic ischemia: etiology, pathogenesis, clinical presentation and management. Minerva Urol Nefrol. 2014;66(2):127-137.

19. Wang W, Zhang N, Zhu XH, et al. Involvement of TL1A and DR3 in induction of ischaemia and inflammation in urinary bladder dysfunction in the elderly. Mol Med Rep. 2012;6(2):434-438.
20. Wada N, Matsumoto S, Kita M, Hashizume K, Kakizaki H. Improvement of overactive bladder symptoms and bladder ischemia with dutasteride in patients with benign prostatic enlargement. Low Urin Tract Symptoms. 2015;7(1):37-41.

21. Hossini AM, Quast AS, Plötz M, et al. PI3K/AKT signaling pathway is essential for survival of induced pluripotent stem cells. PLoS One. 2016;11(5): 0154770

22. Zhou L, Luan H, Dong X, LiY. Activation of the PI3K/Akt and MAPK signaling pathways antagonizes adriamycin-induced HL-60 leukemia cell apoptosis. Mol Med Rep. 2010;3(4):641-644.

23. Itoh K, Wakabayashi N, Katoh Y, et al. Keap1 represses nuclear activation of antioxidant responsive elements by Nrf2 through binding to the aminoterminal Neh2 domain. Genes Dev. 1999;13(1):76-86.

24. Kwon J, Han E, Bui CB, et al. Assurance of mitochondrial integrity and mammalian longevity by the p62-Keap1-Nrf2-Nqo1 cascade. EMBO Rep. 2012;13(2):150-156.

25. Seibenhener ML, Du Y, Diaz-Meco MT, Moscat J, Wooten MC, Wooten MW. A role for sequestosome 1/p62 in mitochondrial dynamics, import and genome integrity. Biochim Biophys Acta. 2013;1833(3):452-459.

26. Indo HP, Davidson M, Yen HC, et al. Evidence of ROS generation by mitochondria in cells with impaired electron transport chain and mitochondrial DNA damage. Mitochondrion. 2007;7(1-2):106-118.

27. Haynes CM, Fiorese CJ, Lin YF. Evaluating and responding to mitochondrial dysfunction: the mitochondrial unfolded-protein response and beyond. Trends Cell Biol. 2013;23(7):311-318.

28. Athale J, Ulrich A, Macgarvey NC, et al. Nrf2 promotes alveolar mitochondrial biogenesis and resolution of lung injury in Staphylococcus aureus pneumonia in mice. Free Radic Biol Med. 2012;53(8):1584-1594.

29. Hota SK, Barhwal K, Singh SB, Sairam M, Ilavazhagan G. NR1 and GluR2 expression mediates excitotoxicity in chronic hypobaric hypoxia. J Neurosci Res. 2008;86(5):1142-1152.

30. Kim SK, Joe Y, Zheng M, et al. Resveratrol induces hepatic mitochondrial biogenesis through the sequential activation of nitric oxide and carbon monoxide production. Antioxid Redox Signal. 2014;20(16):2589-2605.

31. Ushio-Fukai M, Alexander RW, Akers M, et al. Reactive oxygen species mediate the activation of Akt/protein kinase B by angiotensin II in vascular smooth muscle cells. J Biol Chem. 1999;274(32):22699-22704.

32. Martindale JL, Holbrook NJ. Cellular response to oxidative stress: signaling for suicide and survival. J Cell Physiol. 2002;192(1):1-15.

33. Cantley LC. The phosphoinositide 3-kinase pathway. Science. 2002;296(5573):1655-1657.

34. Fan CD, Lum MA, Xu C, Black JD, Wang X. Ubiquitin-dependent regulation of phospho-AKT dynamics by the ubiquitin E3 ligase, NEDD4-1, in the IGF-1 response. J Biol Chem. 2012;288(3):1674-1684.

35. Furuyama T, Nakazawa T, Nakano I, Mori N. Identification of the differential distribution patterns of mRNAs and consensus binding sequences for mouse DAF-16 homologues. Biochem J. 2000;349(pt 2):629-634.
Research and Reports in Urology

\section{Publish your work in this journal}

Research and Reports in Urology is an international, peer-reviewed, open access journal publishing original research, reports, editorials, reviews and commentaries on all aspects of adult and pediatric urology in the clinic and laboratory including the following topics: Pathology, pathophysiology of urological disease; Investigation and treatment of

\section{Dovepress}

urological disease; Pharmacology of drugs used for the treatment of urological disease. The manuscript management system is completely online and includes a very quick and fair peer-review system, which is all easy to use. Visit http://www.dovepress.com/testimonials.php to read real quotes from published authors. 